\title{
COVID-19 Are these Viruses Homogenous in Virulence An Engineering perspective
}

\author{
Ho Teck Tuak, PhD. \\ Keywords: COVID-19, High Fever, Shortness of Breath, Mild Symptoms, No Symptoms, Virulence
}

When large droplets of these Viruses are expelled into the surrounding, they are likely to be among those most virulent and strong. These Viruses are the ones most likely able to make the journey to the recipient lung tissue and cause typical symptoms for COVID-19: High Fever with Shortness of Breath.

Another pathway for transmission is through skin absorption. These Viruses entering the skin surface is likely to create unusual patch on the skin and may even show up as tiny dots under high resolution microscope. This patch may not be typical red because the immune system does not yet able to recognize it. Once entering the skin surface, the stronger ones head for the lung tissue in a journey that may take a few days. The less strong ones remain as residue in the skin tissue and other tissues along the journey. The skin and other tissues along the journey do excretions throughout the day. Excretions from skin surface include sweat. This Virus may be included in the sweat and is highly contagious. It can be transmitted to another potential patient. It is likely suspending in some form of viscous solution with water retention ability to remain sticking on surfaces and not evaporate immediately. In colder climate, such solution may remain longer time on the surface before evaporating while in more tropical climate, such solution may remain shorter time before evaporating. However, as this type of remaining Virus is less strong, it is likely to only cause other typical mild or no symptoms to the receiver because such Virus is unlikely to be able to be strong enough to reach the receiver lung tissue. As this Virus is not yet recognizable by the immune system, whether the Virus is more or less virulent depends on the strength of the Virus itself. Whether this Virus will be strong enough eventually to reach the receiver lung tissue depends on how close the entry site is to the lung tissue. 